\title{
Periodically Slotted Coupled Mode Leaky Coaxial Cable with Enhanced Radiation Performance
}

\author{
Zeeshan Siddiqui, Marko Sonkki, Marko Tuhkala and Sami Myllymäki
}

\begin{abstract}
A novel technique to periodically modulate the aperture of a coupled mode leaky coaxial cable (LCX) is presented in this communication. The surface wave (monofilar mode) over the cable is modulated by periodically slotted aperture, converting it to a leaky wave. This leaky wave due to periodic modulation complements the leaked bifilar mode, thus enhancing the radiation efficiency of the LCX. The numerical results obtained through CST simulation are presented to prove the co-existence of a complex surface wave and a leaky wave over the surface of periodically modulated cable. As a proof of concept, a periodically slotted LCX operating at $4.5 \mathrm{GHz}$ is designed and fabricated. Parametric studies are performed to observe the influence of the key parameters on the radiation performance. The measured results reasonably agree with the simulations.
\end{abstract}

Index Terms-Indoor communication system, frequency beamscanning antenna, leaky coaxial cable ( $L C X)$, leaky wave antenna

\section{INTRODUCTION}

Conventionally leaky coaxial cable (LCX) has been extensively used in mines, railway and subway tunnels as a preferred choice for antenna [1]-[3]. The uniform radiation nature of the LCX very well suits the coverage requirements of the long and shallow areas. In recent years, there is a renewed interest in exploring new applications of leaky coaxial cable (LCX). The current research and development directions are focusing on higher frequencies and wider bandwidths with applications ranging from sensors to wireless LAN access systems [4]. The LCX dual nature, that is simultaneous guiding and radiation of electromagnetic wave, and simpler installation makes it an attractive choice for indoor coverage. Initially, it was reported as a base-station antenna for in-building communication systems for improving the radio coverage [5]. More recently, its application in multiple-input and multiple-output (MIMO) communication systems was explored for enhancing both the coverage and capacity of the indoor wireless systems. An experimental study of MIMO performance at $1.8 \mathrm{GHz}$ using LCXs in a tunnel environment was presented in [6]. The feasibility of using LCX in MIMO communication at 2.4 and $5 \mathrm{GHz}$ was demonstrated in [7].

Based on the radiation mechanism, LCX may be categorized in two types, coupled mode and radiating mode, depending on the slots' spacing. The coupled mode cable, subject of this paper, has a continuous aperture. While the radiating mode cable is a periodic structure where slot spacing is a function of operating wavelength. Although it has better radiation performance in comparison to the coupled mode cable, still it might not be enough improvement in all the cases. In addition, it has higher longitudinal attenuation and the received signal might fluctuate at different locations along the length, due to its inherent array like radiation mechanism [8].

The limited and uniform radiation nature of the LCX offers no flexibility for the coverage requirements of complex indoor propagation environment, for example large buildings having offices,

This work was supported in part by the Finnish Funding Agency for Technology and Innovation (Tekes) and its industrial partners Prysmian Group, Elcoflex and Premix under Smart Wireless Cable project, and in part by the Academy of Finland 6Genesis Flagship (grant no. 318927).

Zeeshan Siddiqui and Marko Sonkki are with the Centre for Wireless Communications (CWC), University of Oulu, Oulu FI-90014, Finland (e-mail: zeeshan.siddiqui@oulu.fi

Marko Tuhkala and Sami Myllymäki are with the Microelectronics Research Unit, University of Oulu, Oulu FI-90014, Finland. corridors and halls. In similar scenarios, an LCX alone cannot be a viable solution from the radio coverage aspect. The spatial coverage requirement in a building requires adjustable radiation performance of the cable. Earlier, a simple solution was presented by introducing a manual mechanism to activate or deactivate the radiating slots; it provided a control only to avoid unwanted radiation [9]. Another solution was proposed by loading the cable with periodic metallic patches. The patches were excited by the surface wave over the outer conductor, causing an increase in radiation efficiency of the patch loaded LCX [10].

In this paper, a novel solution with wide bandwidth, better control over the efficiency and the direction of maximum radiation is proposed for LCX based indoor communication systems [11]. The aperture of coupled mode LCX is periodically modulated to transform the surface wave (monofilar mode) into a leaky wave. This leaky wave is an addition to the leaked bifilar mode (Section II). It adds the feature of directional radiation to the LCX along with the enhancement of its radiation efficiency. The leaky wave direction and magnitude can be controlled according to the theory of spatial harmonics [12]. The spatial coverage capability of the ordinary LCX installation can be improved by complementing it with the segments of the modulated LCX along its length, according to the requirement. For example, an indoor installation scenario of an LCX is shown in Fig. 1(a). There might be coverage problem with ordinary LCX, especially in the hall area. This can be mitigated by introducing a section of periodically modulated LCX across the normal LCX.

In recent years, much research effort has been made to develop lowcost, high gain leaky wave antennas for different applications. A broadband and high gain endfire antenna is demonstrated for longdistance wireless applications [13]. In [14] and [15], endfire antennas with high gain are presented for large metallic platforms. The proposed technique of periodic aperture modulation can also be adopted to transform the LCX into a novel, high gain, frequency beam-scanning leaky wave antenna as shown in Fig. 1(b). Waveguide transitions can be saved by replacing the conventional waveguide antennas with the presented periodically modulated LCX. The proposed leaky wave antenna is a good trade-off between the electrical performance and other merits like the compactness, cost, ruggedness and the power handling ability. It has an added advantage of simpler installation in distributed antenna systems (DAS) with coaxial cables.

This paper is organized as follows. Section II presents the design and analysis of the proposed periodically modulated LCX. Prototype fabrication and the measured results are discussed in Section III. Finally, the paper is concluded in Section IV.

\section{Design AND ANALysis}

At first, a standard 7/8 inch, 50-ohm coaxial cable was modeled for the proposed design with a cut-off frequency of $5.1 \mathrm{GHz}$. The inner and outer conductor diameter was 9.0 and $23.2 \mathrm{~mm}$ respectively. The dielectric foam confined between conductors was cellular polyethylene with dielectric constant of 1.25 and loss tangent of 0.0002 . The thickness of the outer conductor copper foil was $0.15 \mathrm{~mm}$. The outer jacket, usually made of polyethylene, was excluded from the simulation model as it has minimal effect on the electrical performance of the cable [8]. Circumferential slots were etched over 


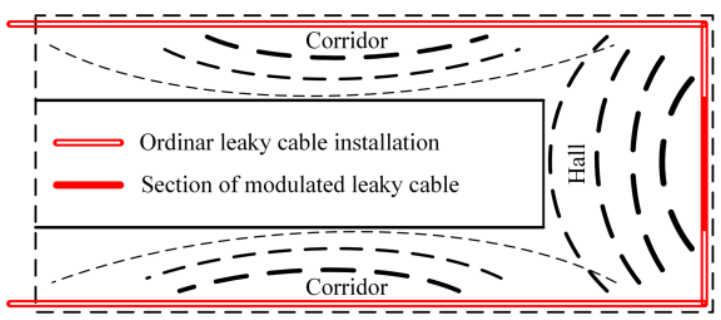

(a)

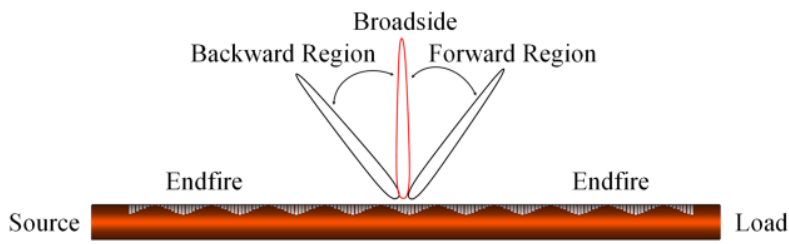

(b)

Fig. 1. Application scenarios for the proposed technique (a) Periodically modulated leaky coaxial cable complementing the ordinary leaky cable to enhance the coverage of indoor building environment (b) Leaky cable transformed into a high gain beam-scanning antenna

the outer conductor with a spacing of $1 \mathrm{~mm}, 0.015 \lambda$ at $4.5 \mathrm{GHz}$ to realize a coupled mode LCX.

The problem of wave propagation along the LCX has been solved by using different analytical and numerical techniques [16], [17]. It is established that two distinct guided modes are supported by the LCX in free space. Both the modes co-exist at all the operating frequencies with different wave velocities. The bifilar or coaxial mode is mostly confined between the center and outer conductor of the coaxial cable while some of the energy leaks outside from the slots; the leakage is utilized for the communication purpose. On the contrary, the monofilar mode is spread over the outer side of the outer conductor, similar to Gaubau mode. Eigenmode solver of the CST Microwave studio (MWS) is utilized to plot the electric field distribution of the stated modes in Fig. 2. In the following subsection, the wave number of the waves propagating along the LCX surface are extracted utilizing CST MWS [18].

\section{A. Near Field Analysis of coupled mode LCX}

Electric field probes are placed along the length of LCX to sample the near field in the simulation model as shown in Fig. 3. They are at the center of the aperture with a spacing of $4 \mathrm{~mm}$ between them. The length of simulated structure was kept $12 \lambda$ at $4.5 \mathrm{GHz}$. As discussed earlier for a coupled mode LCX, there exists a leaky wave like bifilar mode and a surface wave like monofilar mode over the surface. The travelling wave over the structure is superposition of these two waves, which can be written as,

$$
E_{z}=A_{s} e^{-j k_{s} y}+A_{l} e^{-j k_{l} y}
$$

where $A_{\mathrm{s}}$ and $A_{1}$ are the amplitudes, $k_{\mathrm{s}}$ and $k_{1}$ are the propagation constant of the surface and leaky wave respectively.

The near field data along the structure length is numerically fitted to Equation (1) utilizing the non-linear, least square curve fitting function of Matlab. In case of the LCX, choice of initial guess for the surface wave is a real number, and a complex number for the leaky wave. The propagation constant of the surface wave and leaky wave can be initially estimated as of free-space and dielectric material of cable, respectively. For better accuracy of the calculated propagation constant, it is recommended to sample the near field outside the cable in case of surface wave, and inside the cable for the leaky wave. However, in the presented case it was sampled outside the LCX to demonstrate the co-existence of the two waves. In Fig. 4, propagation constant for the surface wave (monofilar mode), normalized by free-

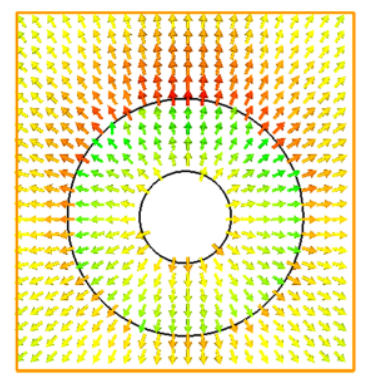

(a)

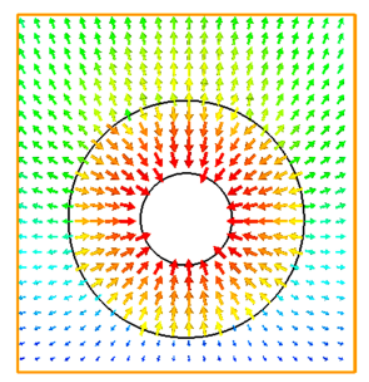

(b)
Fig. 2. (a) Normalized electric field distribution of monofilar mode and (b) bifilar mode at $4.5 \mathrm{GHz}$.

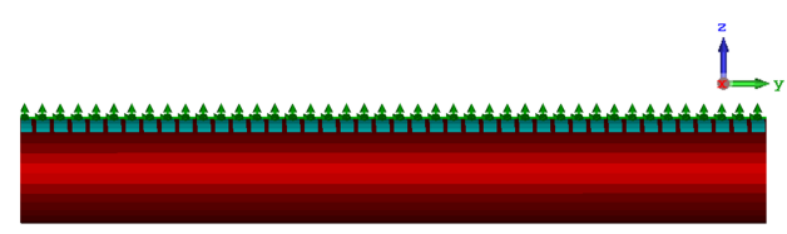

Fig. 3. Section of the LCX with electric field probes over the surface.

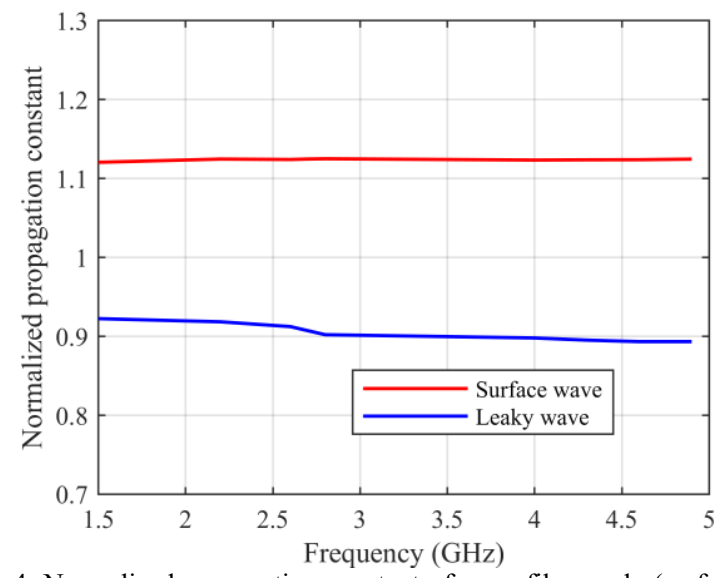

Fig. 4. Normalized propagation constant of monofilar mode (surface wave) and bifilar mode (leaky wave) estimated by near-field analysis.

space wave propagation constant, $k_{0}$, and the leaky wave (bifilar mode), normalized by wave propagation constant in cable dielectric material, $k_{1}$, are calculated utilizing the above discussed technique. It is demonstrated that a surface wave and leaky wave co-exist at the surface of the designed LCX. The speed of bifilar mode (leaky wave) is faster than corresponding speed of light in cable dielectric material. On the other hand, monofilar mode (surface wave) is slower than the wave in free space.

\section{B. Design of Periodically slotted LCX}

A periodically slotted LCX operating at $4.5 \mathrm{GHz}$ was designed and simulated utilizing CST MWS. The bounded or surface wave radiates because of the periodicity along the guiding structure. The field above the structure can be expanded in infinite spatial harmonics, the propagation constant is characterized by, $\beta_{\mathrm{n}}=\beta_{\mathrm{o}}+2 n \pi / a$, where $\beta_{\mathrm{n}}$ is the propagation constant of nth spatial harmonic, $\beta_{\mathrm{o}}$ is the propagation constant of the unmodulated structure, and $a$ is the modulation period. For $n=-1$, harmonic, it can be written as, $\beta_{-1}=\beta_{0}-2 \pi / a$. The beam direction, $\theta$ can be calculated by the following [12],

$$
\theta=\sin ^{-1} \frac{\beta_{-1}}{k_{o}}
$$

The period of the unit cell was chosen to be $64 \mathrm{~mm}$, corresponding to one free-space wavelength at $4.5 \mathrm{GHz}$. A single period had 16 


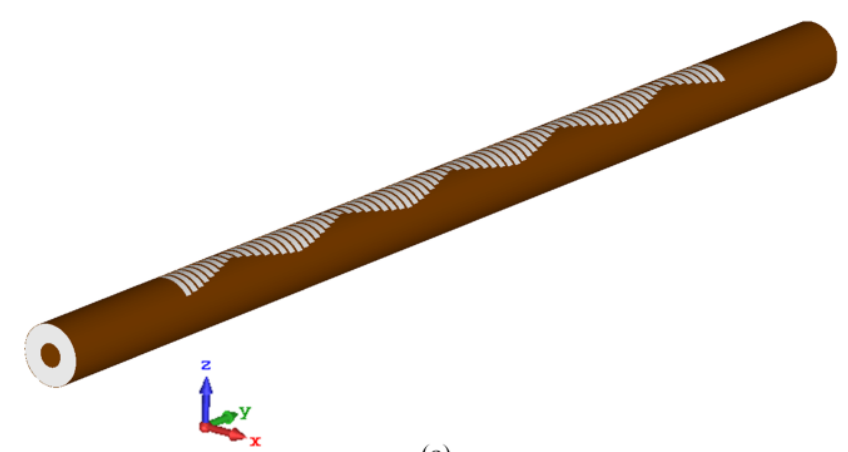

(a)

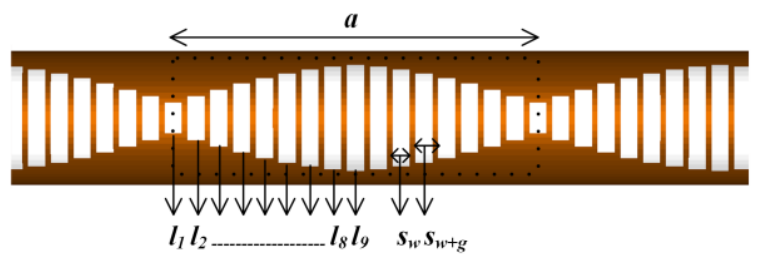

(b)

Fig. 5. (a) CST Microwave Studio 3D simulation model showing perspective view of the periodically slotted leaky coaxial cable. (b) Zoomed section of the simulation model.

TABLE 1

DIMENSIONS OF THE PERIODICALLY MODULATED LCX

\begin{tabular}{c|cccccc}
\hline \hline Parameters & $l_{1}$ & $l_{2}$ & $l_{3}$ & $l_{4}$ & $l_{5}$ & $l_{6}$ \\
\hline Values $(\mathrm{mm})$ & 21.4 & 20.5 & 19.0 & 17.2 & 15.0 & 12.7 \\
\hline Parameters & $l_{7}$ & $l_{8}$ & $l_{9}$ & $s_{\mathrm{W}}$ & $s_{\mathrm{w}+\mathrm{g}}$ & $a$ \\
\hline Values $(\mathrm{mm})$ & 10.3 & 7.9 & 5.5 & 3.0 & 4.0 & 64.0 \\
\hline
\end{tabular}

circumferential slots around the cable with a fixed slot width of $3 \mathrm{~mm}$; the gap between slots was $1 \mathrm{~mm}$. The angular slot aperture was modulated according to the following Equation (3),

$$
l(y)=l_{i}\left(1+\operatorname{MCos}\left(\frac{\pi y}{a}\right)\right)
$$

where $l$ is the slot aperture, $l_{\mathrm{i}}$ is the initial slot aperture, $M$ is the modulation period, and $a$ is the period. The modulation factor, $M$, mainly controlling the leakage rate was optimized by iterative simulations to achieve the desired radiation performance. The simulation model is shown in Fig. 5.

In the following subsection, a parametric study is presented to have better understanding of the wave-guiding and radiation phenomenon of periodically modulated LCX.

\section{Parametric Study}

To study the effect of periodicity, $a$, it was simulated at two different values. It was selected $64 \mathrm{~mm}$ for the first case and increased to $120 \mathrm{~mm}$ for the second case only by changing the slot width and gap proportionally. The simulated length of the structure was 10 periods in both the cases. All the other parameters were kept same (Table 1). The directional radiation is due to the periodic modulation, it mainly depends on the period, $a$, and calculated by Equation 2. The propagation constant of the unmodulated structure, $\beta_{0}$, was estimated using the near field technique discussed earlier in section II A. The calculated and simulated direction of maximum radiation are compared in Fig. 6. A good agreement between them can be observed in both the cases, except to a slight discrepancy at higher frequencies in case 1. The modulation factor also influences the direction of maximum radiation. It is kept constant at 2.4 in both the cases, only to observe the effect of periodicity.

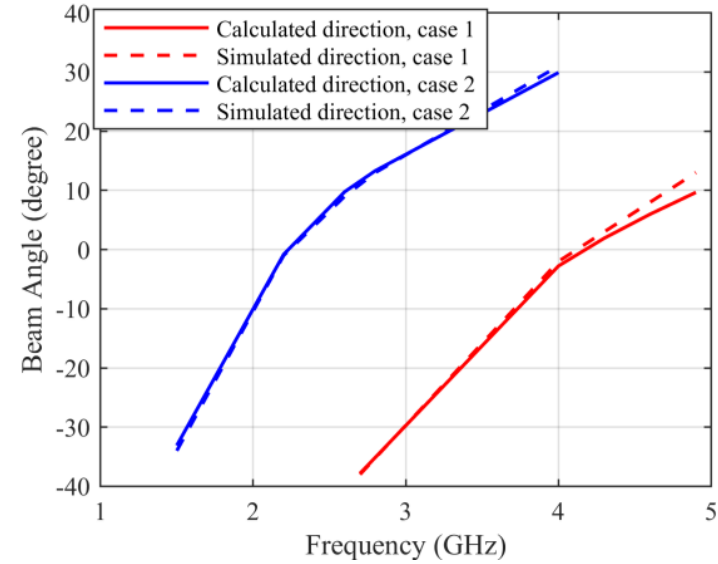

Fig. 6. Comparison of calculated and simulated beam direction to study the effect of periodicity, $a$. In case $1, a=64 \mathrm{~mm}$, and case $2, a=120 \mathrm{~mm}$.

Table 2 presents the extracted surface wave and leaky wave parameters with different modulation factor, $M$, utilizing the discussed near field technique. In the first case with no modulation, it can be observed from the Table 2 that the surface wave has real component and only the leaky wave due to slot aperture is contributing in radiation. The amplitude of leaky wave is low, as the slot aperture is small. As the modulation is increased, the surface wave transforms into complex, due to radiation. With modulation factor, $M=1$, the conversion is not significant, but as it is further increased, a noticeable increase in the normalized attenuation constant $\left(\alpha / k_{\mathrm{o}}\right)$ of the complex surface wave can be noticed. On the other hand, leaky wave normalized attenuation $\left(\alpha / k_{1}\right)$ is decreasing as the mean aperture is increasing due to increment in $M$. It can be observed with the amplitudes of surface and leaky waves that the strength of former is decreasing, and the latter is increasing due to higher modulation factor. It means that there is more radiation from both the sources of radiation with the increase of mean aperture. The $M$ do not have a significant effect on the normalized propagation constant $\left(\beta / k_{1}\right)$ of the leaky wave. However, $\left(\beta / k_{0}\right)$ of the surface wave is gradually increased with the increment of $M$, it shows that the beam direction also depends on modulation factor to a lesser extent.

The effect of $M$ on the radiation pattern is presented in Fig. 7. With $M=0$, it is an ordinary LCX. The gain is very low, and the pattern is like an end-fire antenna. All the radiation is due to the leaky wave from the slot aperture. As $M$ is increased to 1, the gain is slightly improved, but the end fire lobe is still dominant over the directional radiation due to modulation. The gain is gradually increased and the directional radiation due to periodic modulation became dominant over the end fire radiation due to incremental $M$. However, the endfire lobe is still relatively high as the radiation due to the leaky wave also increases with the mean aperture increment. It is important to note here that the power in the end lobe appears to increase again if the modulation factor is higher than the optimum value, as shown in the comparison of plots for $M=2.5$ and $M=3.0$.

The effect of $M$ on the S-parameters is shown in Fig. 8. The $\left|\mathrm{S}_{21}\right|$ plot shows the increase in efficiency with the increment of $M$, if all the other negligible losses are ignored. The mean aperture increases due to higher $M$, deteriorating the matching as shown in the $\left|\mathrm{S}_{11}\right|$ plot. Around $2 \mathrm{GHz}$ there is a stop band especially at high $M$, this is due to the backward wave in end fire direction.

The influence of the electrical length or the number of periods of modulated structure over the radiation pattern is observed in Fig. 9. It is obvious from the graph that the gain of the antenna increases, and the beam width decreases with the increase in length. It can be observed from the graph that the antenna can be optimized as a beam 
TABLE 2

Extracted Surface Wave And Leaky Wave Parameters Versus VARYING MODULATION FACTOR AT $4.5 \mathrm{GHz}$

\begin{tabular}{lcccccc}
\hline \hline & \multicolumn{3}{c}{ Surface wave } & \multicolumn{3}{c}{ Leaky wave } \\
\hline$M$ & $\alpha / k_{o}$ & $\beta / k_{o}$ & $\left|A_{s}\right|$ & $\alpha / k_{1}$ & $\beta / k_{l}$ & $\left|A_{l}\right|$ \\
\hline 0 & 0.0 & 1.1236 & 3.5159 & 0.0185 & 0.8923 & 0.0629 \\
\hline 1 & 0.0003 & 1.1321 & 3.3002 & 0.0158 & 0.8971 & 0.2521 \\
\hline 2 & 0.0018 & 1.1640 & 2.6387 & 0.0101 & 0.9092 & 0.8932 \\
\hline 2.5 & 0.0046 & 1.2029 & 2.0291 & 0.0077 & 0.9173 & 1.3914 \\
\hline 3 & 0.0230 & 1.3415 & 0.8411 & 0.0041 & 0.8971 & 2.4210
\end{tabular}

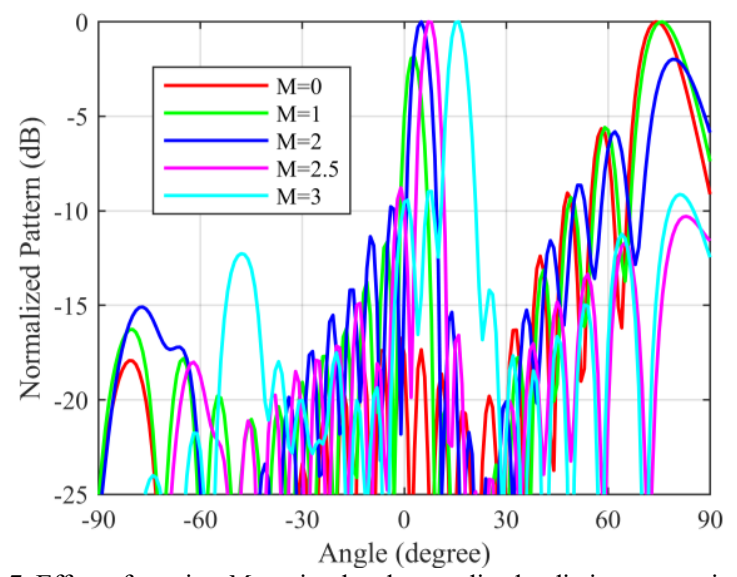

Fig. 7. Effect of varying $M$ on simulated normalized radiation pattern in E-plane (yz-cut) at $4.5 \mathrm{GHz}$.

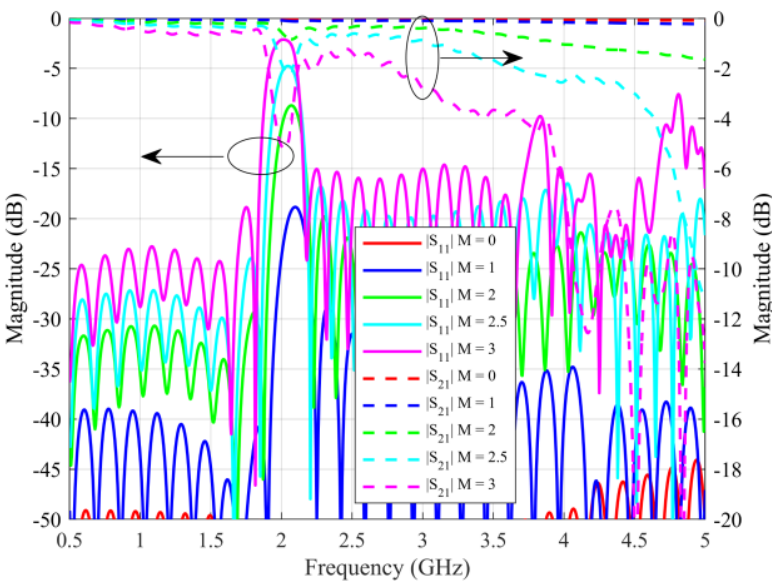

Fig. 8. Effect of varying $M$ on the S-parameters of the LCX.

scanning leaky wave antenna within a length of 10-15 wavelengths by adjusting the modulation factor. Another feature of extended length is the decreased power in the side lobes. This means longer length antennas have better side lobe performance.

The impedance matching is not affected with the increasing length of structure as shown in Fig. 10. Only the stop band due to backward surface wave became prominent with the incremented length. It also shows that the modulated surface wave is the major contributor in a longer length antenna. From the $\left|\mathrm{S}_{21}\right|$ plots it can be observed that the received power at the terminated port decreases, showing the increase in efficiency with the added length.

The periodically modulated LCX can be designed according to the application requirements by judiciously selecting the periodicity, modulation factor and the electrical length of the structure, as observed from the parametric studies. From the application aspect, indoor coverage capability of the ordinary LCX can be enhanced by introducing segments of judiciously designed periodically modulated LCX. Empirical studies of the modulated LCX in any environment

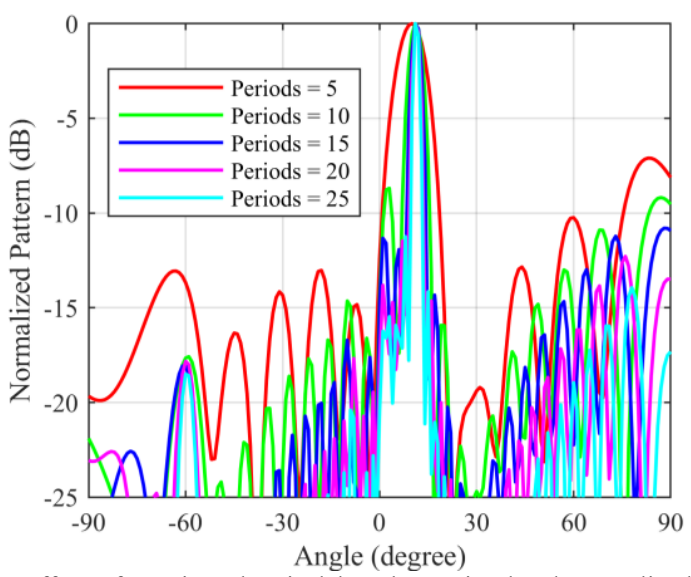

Fig. 9. Effect of varying electrical length on simulated normalized radiation pattern in E-plane (yz-cut) at $4.5 \mathrm{GHz}$.

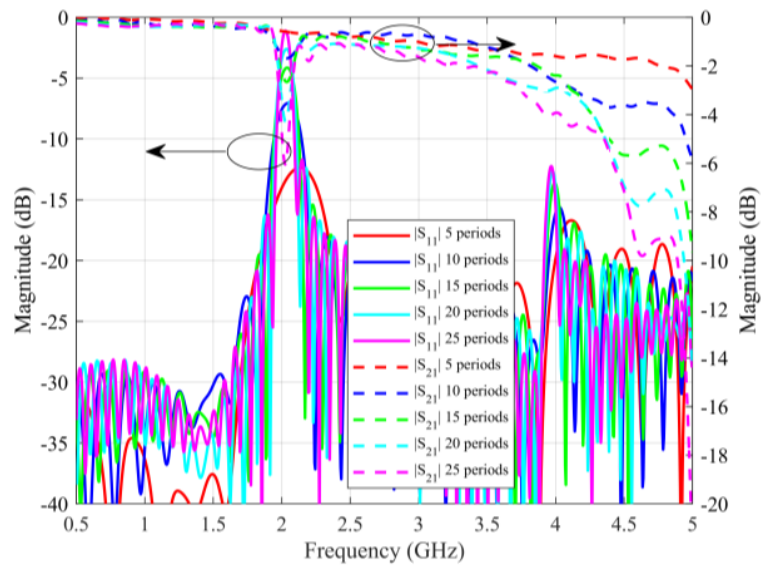

Fig. 10. Effect of varying electrical length on the S-parameters of the LCX.

can assist in selecting the appropriate modulation factor and electrical length. In case of, a beam-scanning leaky wave antenna, the direction of radiation at the operating frequency can be calculated utilizing Equation 2. It can be designed, with electrical length in between 1015 wavelengths, for low cost, rugged applications.

\section{FABRICATION, RESULTS AND DISCUSSION}

\section{A. Prototyping}

To demonstrate the proposed idea and compare the theory to practice, a prototype was fabricated and measured. The prototype had the same dimensions as presented in Table 1. The cable was terminated with a 50-ohm termination to avoid the reflection of the remaining signal. The $\mathrm{N}$-type connector body occupied the space of $60 \mathrm{~mm}$ over the cable at each end. The total length of the prototype had to be limited to $0.45 \mathrm{~m}$, as it is the maximum antenna under test (AUT) dimension allowed by Satimo StarLab antenna measurement system, used for the radiation pattern measurement. The prototype was fabricated by simply replacing the outer conductor of a commercially available LCX by a copper foil with the designed slot pattern. The slots in a plain copper foil were cut through by a laser machine. The fabricated prototype with five periods is shown in Fig. 11.

\section{B. Simulated and Measured Results}

The reflection coefficient, $\left|\mathrm{S}_{11}\right|$ and transmission coefficient, $\left|\mathrm{S}_{21}\right|$ were measured from $500 \mathrm{MHz}$ to $5 \mathrm{GHz}$, utilizing Rohde \& Schwarz ZVB20 vector network analyzer (VNA). The simulated and measured results are presented in Fig. 12. The measured results agree well with 


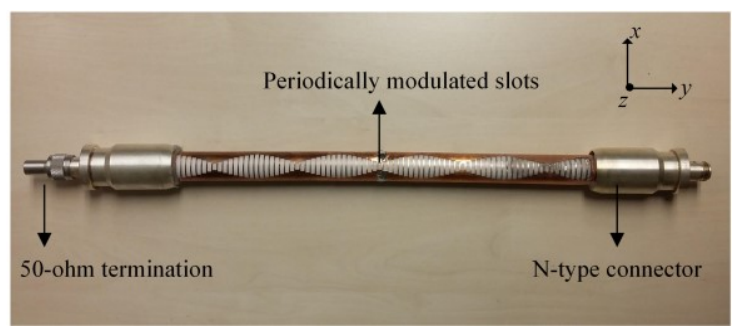

Fig. 11. Fabricated prototype with N-type connectors. Total length of $45 \mathrm{~cm}$, the modulated aperture length is $32 \mathrm{~cm}$.

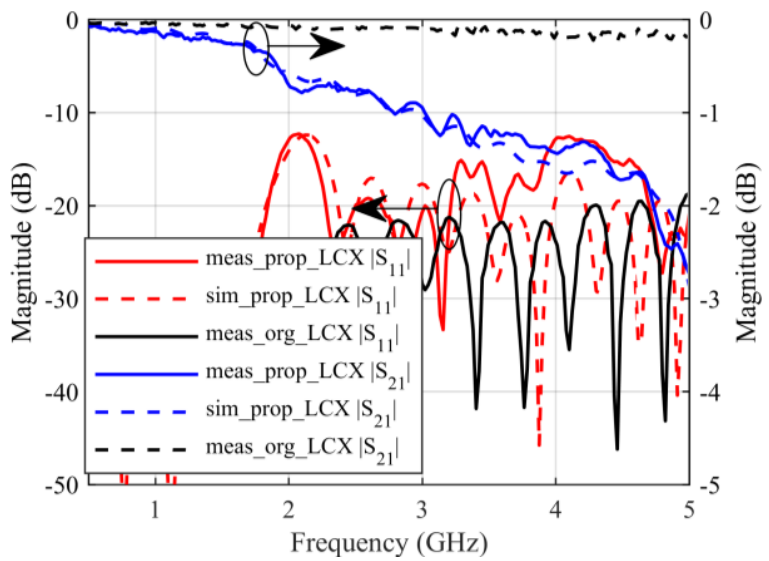

Fig. 12. Comparison of simulated and measured S-Parameters.

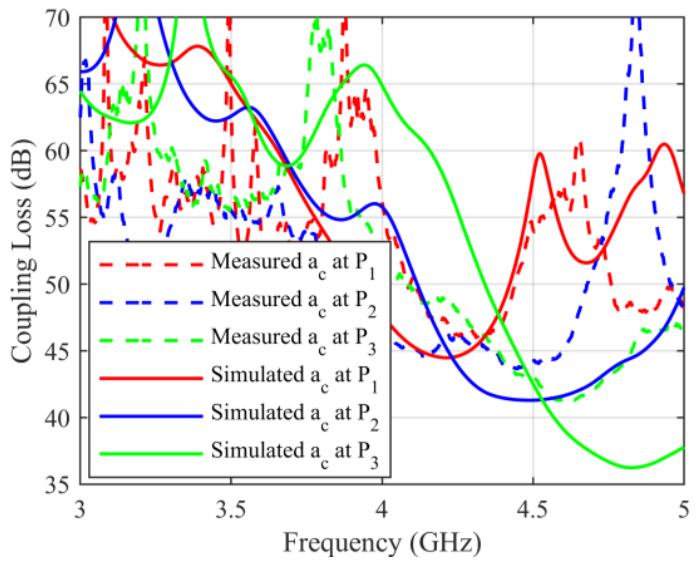

Fig. 13. Comparison of simulated and measured coupling loss.

the simulated ones. At $4 \mathrm{GHz}$, there is higher reflection coefficient due to the reflection of energy back to the structure instead of radiation because of broadside radiation [19]. Overall, the measured $\left|S_{11}\right|$ remains below $-12 \mathrm{~dB}$ over the studied frequency range. The $\left|\mathrm{S}_{21}\right|$ curve indicates the remaining signal at the terminated end of the designed LCX. It shows only around half of the energy is radiated while the remaining is absorbed at the port. This is due to the reduced length of the prototype, due to restriction of pattern measurement facility. If the minimal conductor and dielectric losses are ignored, the radiation efficiency of the structure can be calculated by $1-\left|\mathrm{S}_{11}\right|^{2}-\left|\mathrm{S}_{21}\right|^{2}$. It can be increased arbitrarily by increasing the electrical length of the structure. It is also evident from low $\left|\mathrm{S}_{11}\right|$ and high $\left|\mathrm{S}_{21}\right|$ that the structure behaves more like an ordinary LCX at frequencies below $2 \mathrm{GHz}$, as the modulation period is sufficiently small. The measured results are also compared with a commercially available standard LCX, RFX 7/8"-50. The dielectric material and the cable physical dimensions are same as of the proposed structure. The uniform aperture with slot length of $10 \mathrm{~mm}$ and gap between slots of $0.5 \mathrm{~mm}$ is the only difference. The impedance matching of the original cable is better than the prototype as the slot aperture of is much lower

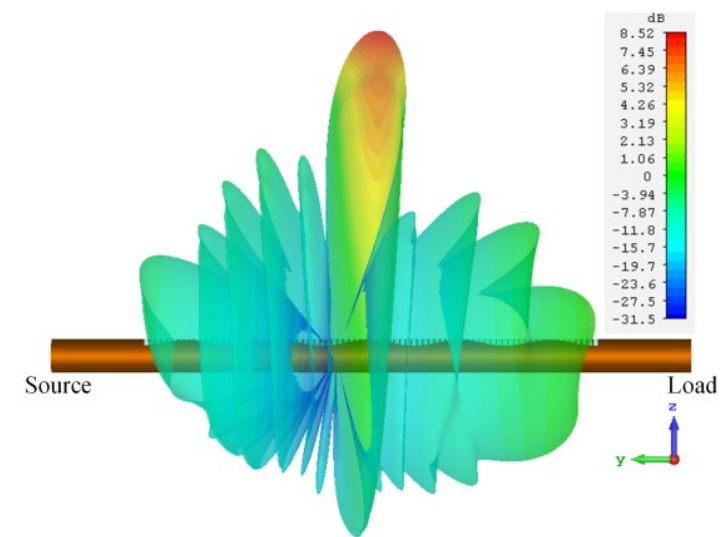

Fig. 14. Simulated 3D realized gain pattern at $4.5 \mathrm{GHz}$.

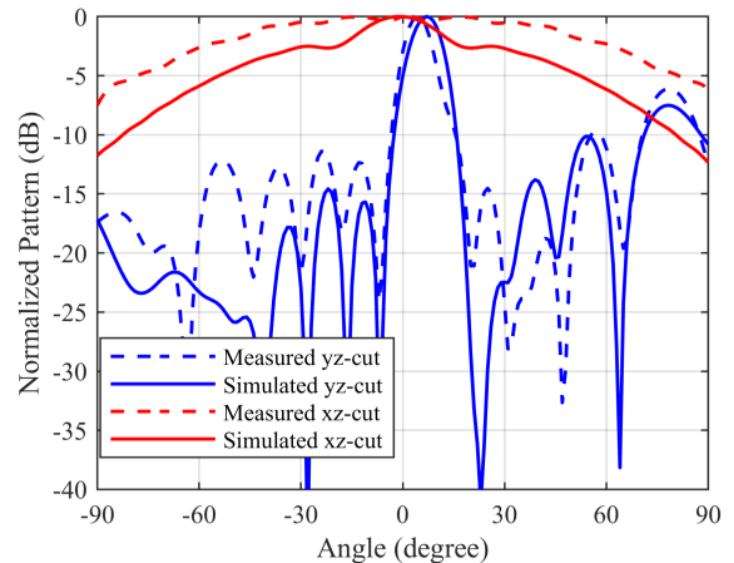

Fig. 15. Measured and simulated radiation pattern in E-plane (yz-cut) and H-plane ( $x z$-cut).

than the mean aperture of the prototype. The $\left|\mathrm{S}_{21}\right|$ of the original LCX is around $-0.2 \mathrm{~dB}$, showing a poor radiation efficiency. The difference in $\left|\mathrm{S}_{21}\right|$ of the proposed and commercial cable at lower frequencies, below $2 \mathrm{GHz}$, is only due to the larger mean aperture of the proposed LCX. The commercial cable is purposely made with small aperture, as it is generally used to cover long tunnel like areas.

The LCXs are generally classified by their coupling loss, $a_{\mathrm{c}}$. It is calculated as, $a_{\mathrm{c}}=-10 \log \left(P_{\mathrm{r}} / P_{\mathrm{t}}\right)$, where $P_{\mathrm{r}}$ is the received power, and $P_{\mathrm{t}}$ is the input power to the cable. In simulation, the $P_{\mathrm{t}}$ was kept at 0.5 watt, and the $P_{\mathrm{r}}$ was calculated by the recorded electric field strength, $E$, utilizing the empirical relation, $P_{\mathrm{r}}=0.13 \lambda^{2} .|E|^{2} / 120 \pi[8]$. Three electric field probes were longitudinally placed in the simulation model, to record the $E$. The first one, $\mathrm{P}_{1}$, at the feeding point, the second, $\mathrm{P}_{2}$, in the middle, and the last, $\mathrm{P}_{3}$, at termination of the LCX. The $a_{\mathrm{c}}$ was measured following the method described in [20]. A wide band Vivaldi antenna [21] was used as a receiving antenna, due to unavailability of standard dipoles at the measured frequencies. The gain of the Vivaldi antenna was compensated from the measured values. Fig. 13 shows the comparison of the simulated and measured coupling loss values of the designed LCX. The simulated and measured results follow the same trend, showing a significant decrease in coupling loss compared to the LCX (without periodic modulation), which has minimum simulated loss of $65 \mathrm{~dB}$. The coupling loss of commercial LCX is presented statistically in the data sheet with $10 \mathrm{~dB}$ tolerance. Its highest measurement frequency is $2.6 \mathrm{GHz}$, with the minimum coupling loss of $63 \mathrm{~dB}$ [22].

The $3 \mathrm{D}$ realized gain pattern at $4.5 \mathrm{GHz}$ is shown in Fig. 14. The measured and simulated normalized radiation patterns in $y z$-plane and $x z$-plane at $4.5 \mathrm{GHz}$ are presented in Fig. 15. The beam-width is wide in $x z$-plane as compared to the $y z$-plane. The simulated and measured normalized radiation pattern in the $y z$-plane, from $4.5 \mathrm{GHz}$ to $4.9 \mathrm{GHz}$ 


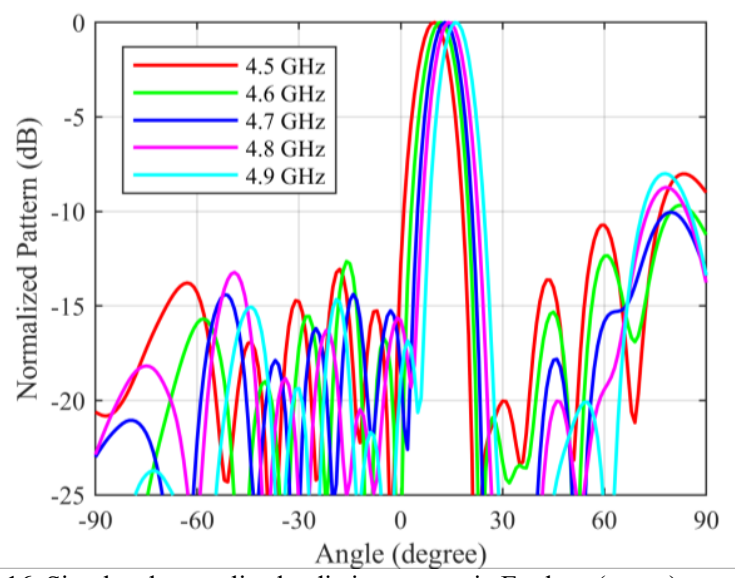

Fig. 16. Simulated normalized radiation pattern in E-plane ( $y z$-cut)

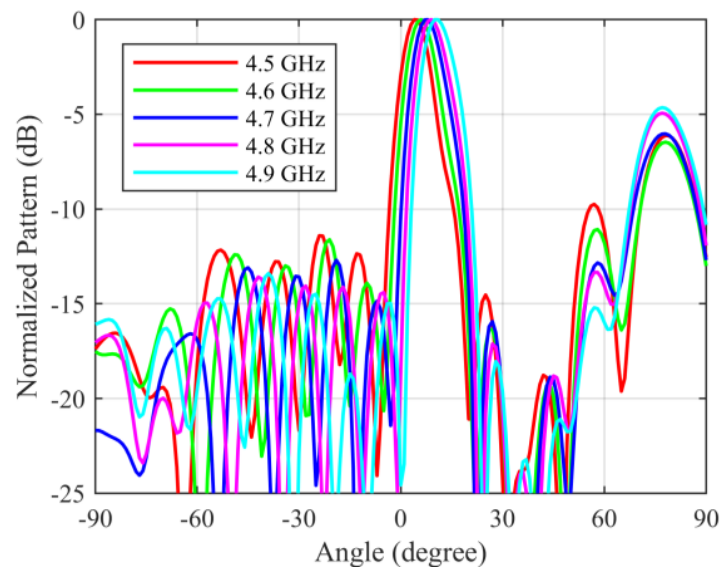

Fig. 17. Measured normalized radiation pattern in E-plane (yz-cut)

are shown in Figs. 16 and 17. It is good to notice that the side lobe level is higher in the designed prototype in comparison to the periodic leaky wave antennas as the designed length was deliberately kept shorter for the sake of pattern measurements.

The minor discrepancies between the simulated and measured results are due to inaccuracies in the prototype fabrication and measurement. In fabrication, the manual rolling and the soldering left small air gaps between the copper foil and the dielectric rod. It reduced the effective dielectric constant of the prototype. The small gaps were random, and it is not simple to model them in the simulations. The prototype is slightly curved due to the softness of used dielectric material. This increases the disagreement between the simulated and measured beam directions.

\section{CONCLUSION}

A novel solution to enhance the radiation efficiency of coupled mode LCX is presented in this paper. The proposed structure has two sources of radiation, the first owing to leakage of coaxial mode from the slots, and the second due to the transformation of surface wave due to periodic modulation which enhances the overall radiation performance. The radiation due to the periodic modulation of surface wave is directional in contrast with the radiation due to leakage. As a proof of concept, a prototype is designed, fabricated and measured at 4.5 GHz. The measured results reasonably agree with the simulated one. From the application perspective, the presented structure can complement the ordinary LCX installation to improve its coverage performance in a complex indoor propagation environment. In addition, the proposed technique can also be utilized to design a frequency beam scanning, high gain antenna based on coaxial cable.

\section{ACKNOWLEDGMENT}

The authors are grateful to Veli-Pekka Moilanen for his assistance in the prototype fabrication.

\section{REFERENCES}

[1] D. Seidel and J. Wait, "Role of controlled mode conversion in leaky feeder mine communication systems," IEEE Trans. on Antennas Propag., vol. 26, no.5, pp. 690-694, Sep. 1978.

[2] K. Ishizu, M. Kuroda and H. Harada, "Bullet-train network architecture for broadband and real-time access," in Proc.12th IEEE Symposium on Computers and Communications, Jul. 2007, pp. 241-248.

[3] D. Seidel and J. Wait, "Mode conversion by tunnel nonuniformities in leaky feeder communication systems," IEEE Trans. on Antennas Propag., vol. 27, no. 4, pp. 560-563, Jul. 1979.

[4] Y. Hou, J. Zhu, S. Denno and M. Okada, "Capacity of 4-by-4 MIMO channel using one composite leaky coaxial cable with user position information." IEEE Trans. on Veh. Tech., vol. 68, no. 11, pp. 1104211051, Nov. 2019.

[5] A. J. Motley and D. A. Palmer, "Reduced long-range signal reception with leaky feeders," Electronics Lett., vol. 19, no.18, pp. 714, 1983.

[6] Yiming Wu et al, "An Experimental Study of MIMO Performance Using Leaky Coaxial Cables in a Tunnel," IEEE Antenna Wireless Propag. Lett., vol. 16, pp. 1663-1666, 2017.

[7] Yafei Hou et al, "Capacity Evaluation of MIMO Channel With One Leaky Coaxial Cable Used as Two Antennas Over Linear-Cell Environments," IEEE Trans. on Veh. Tech., vol. 66, no. 6, pp. 4636-4646, Jun. 2017.

[8] J. H. Wang and K. K. Mei, "Theory and analysis of leaky coaxial cables with periodic slots," IEEE Trans. on Antennas Propag., vol. 49, no.12, pp. 1723-1732, Dec. 2001.

[9] A. Henrik, J. Berg, and J. Medbo, "Leaky coaxial cable having radiation slots that can be activated or deactivated," U.S. Patent 9431716, 30 Aug. 2016.

[10] J. H. Wang, "Leaky coaxial cable with adjustable coupling loss for mobile communications in complex environments," IEEE Microw. Wireless Components Lett., vol. 11, no.8, pp. 346-348, Aug. 2001.

[11] Z. Siddiqui, A. Radwan, M. Sonkki, M. Tuhkala, and S. Myllymäki "A leaky coaxial cable antenna based on sinusoidally-modulated reactance surface," in Proc. Progress In Electromagnetics Research Symposium Spring (PIERS), May 2017, pp. 3887-3890.

[12] F. Monticone and A. Alu, "Leaky-Wave Theory, Techniques, and Applications: From Microwaves to Visible Frequencies," Proceedings of the IEEE, vol. 103, no.5, pp. 793-821, May 2015.

[13] Y. Hou, Y. Li, Z. Zhang, and Z. Feng. "A Broadband and High-Gain Endfire Antenna Array Fed by Air-substrate Parallel Strip Line," IEEE Trans. on Antennas Propag., vol. 67, no.8, pp. 5717-5722, 2019.

[14] Y. Hou, Y. Li, Z. Zhang, and Z. Feng. "Narrow-width periodic leaky-wave antenna array for endfire radiation based on Hansen-Woodyard condition," IEEE Trans. on Antennas Propag., vol. 66, no. 11 pp. 63936396, Aug. 2018.

[15] Y. Hou, Y. Li, Z. Zhang, and M. F. Iskander. "All-metal endfire antenna with high gain and stable radiation pattern for the platform-embedded application," IEEE Trans. on Antennas Propag., vol. 67, pp. 730-737, Feb. 2019.

[16] E. E. Hassan, "Field solution and propagation characteristics of monofilar-bifilar modes of axially slotted coaxial cable," IEEE Trans. on Microw. Theory and Techn., vol. 37, no. 3, pp. 553-557, March 1989.

[17] D. H. Kim and H. J. Eom, "Mode-matching analysis of axially slotted coaxial cable," IEEE Antenna Wireless Propag. Lett., vol. 4, no. 1, pp. 169-171, 2005.

[18] J. Liu, D. R. Jackson and Y. Long, "Substrate Integrated Waveguide (SIW) Leaky-Wave Antenna with Transverse Slots," IEEE Trans. on Antennas Propag., vol. 60, no.1, pp. 20-29, Jan. 2012.

[19] J. L. Volakis, Antenna Engineering Handbook, 4th ed. New York: McGraw-Hill, 2007.

[20] IEC 61196-4, "Coaxial communication cables - Part 4: Sectional specification for radiating cables," Sep. 2015

[21] M. Sonkki, D. Sánchez-Escuderos, V. Hovinen, E. T. Salonen, and M. Ferrando-Bataller, "Wideband Dual-Polarized Cross-Shaped Vivaldi Antenna," IEEE Trans. on Antennas Propag., vol. 63, no.6, pp. 28132819, Jun. 2015.

[22] Coaxial cable Antenna. Available: https://www.prysmiangroup.com/en. 